\title{
Simulation of Elastic Wave Propagation using Cellular Automata and Peridynamics, and Comparison with Experiments
}

\author{
Vinesh V. Nishawala ${ }^{\mathrm{a}, *}$, Martin Ostoja-Starzewski ${ }^{\mathrm{a}}$, Michael J. Leamy $^{\mathrm{b}}$, Paul N. Demmie ${ }^{\mathrm{c}}$ \\ ${ }^{a}$ Department of Mechanical Science and Engineering, Institute for Condensed Matter Theory and Beckman Institute, \\ University of Illinois at Urbana-Champaign, Urbana, IL 61820, USA \\ ${ }^{b}$ George W. Woodruff School of Mechanical Engineering, Georgia Institute of Technology, 7r1 Ferst Drive, NW, Atlanta, GA \\ 30332-0405, USA \\ ${ }^{c}$ Terminal Ballistics Technology, Sandia National Laboratories, Box 5800 MS 1322, Albuquerque, NM 87185-1322, USA
}

\begin{abstract}
Peridynamics is a non-local continuum mechanics formulation that can handle spatial discontinuities as the governing equations are integro-differential equations which do not involve gradients such as strains and deformation rates. This paper employs bond-based peridynamics. Cellular Automata is a local computational method which, in its rectangular variant on interior domains, is mathematically equivalent to the central difference finite difference method. However, cellular automata does not require the derivation of the governing partial differential equations and provides for common boundary conditions based on physical reasoning. Both methodologies are used to solve a half-space subjected to a normal load, known as Lamb's Problem. The results are compared with theoretical solution from classical elasticity and experimental results. This paper is used to validate our implementation of these methods.
\end{abstract}

Keywords: Peridynamics, Cellular Automata, Lamb's Problem, Wave Propagation, Surface Waves, Validation, bond-based Peridynamics

PACS: 46.40.-f, 62.30.+d

\section{Introduction to Peridynamics}

Peridynamics $(\mathrm{PD})$ is a non-local continuum mechanics theory that avoids spatial derivatives in favor of integro-differential equations that was introduced by Silling in 2000 [1]. The nonlocality of PD means that deformation is influenced by a region, called the horizon, beyond its immediate surrounding.

The governing equation of bond-based PD is given as:

$$
\rho(\boldsymbol{x}) \ddot{\boldsymbol{u}}(\boldsymbol{x}, t)=\int_{H_{x}} \boldsymbol{f}\left(\boldsymbol{u}^{\prime}-\boldsymbol{u}, \boldsymbol{x}^{\prime}-\boldsymbol{x}\right) \mathrm{d} V^{\prime}+\boldsymbol{b}(\boldsymbol{x}, t)
$$

where $\rho$ is the mass density, $\boldsymbol{x}$ is the position, $\boldsymbol{u}$ is the deformation, $t$ is the time, $\boldsymbol{f}$ is the force density between points $\boldsymbol{x}^{\prime}$ and $\boldsymbol{x}$ and $\boldsymbol{b}$ is the body force. The function $\boldsymbol{f}$ is called the pairwise response function. It has units of force per unit volume squared. All material properties are specified through the pairwise response function. The PD interaction between two points is called a bond. The integral in (1) is taken over the volume $H_{x}$, where $H_{x}$ is the set of all points less than some fixed distance $\delta$ from $\boldsymbol{x}$. The distance $\delta$ is called the horizon and $H_{x}$ is called the family of $\boldsymbol{x}$. (1) can be interpreted as a restatement of Newton's Second Law of Motion. In PD, only points within $H_{x}$ are capable of applying a force at $\boldsymbol{x}$.

To find the form of $\boldsymbol{f}$ we begin by defining stretch as:

$$
s\left(\boldsymbol{u}^{\prime}-\boldsymbol{u}, \boldsymbol{x}^{\prime}-\boldsymbol{x}\right)=\frac{\left\|\boldsymbol{y}^{\prime}-\boldsymbol{y}\right\|-\left\|\boldsymbol{x}^{\prime}-\boldsymbol{x}\right\|}{\left\|\boldsymbol{x}^{\prime}-\boldsymbol{x}\right\|}
$$

\footnotetext{
*Corresponding Author, Tel.: + 1217265 0900; fax: + 12172449980

Email address: nishawa2@illinois.edu (Vinesh V. Nishawala)
} 


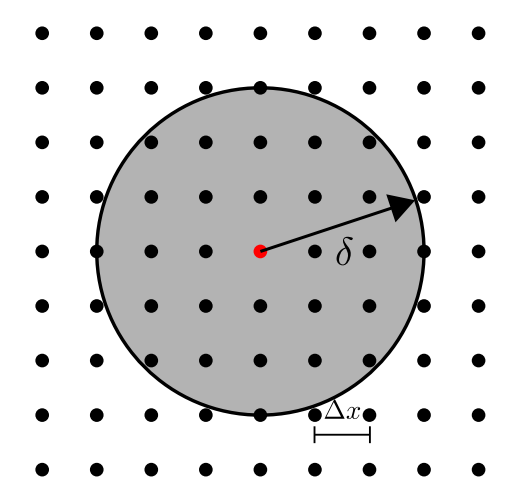

Figure 1: Horizon in Discretized Domain

where $\boldsymbol{y}(\boldsymbol{x}, t)=\boldsymbol{u}(\boldsymbol{x}, t)+\boldsymbol{x}$ and $\boldsymbol{y}^{\prime}(\boldsymbol{x}, t)=\boldsymbol{u}^{\prime}(\boldsymbol{x}, t)+\boldsymbol{x}^{\prime}$, and the double vertical bars denote the 2-norm. The coordinate $\boldsymbol{y}$ is the position of $\boldsymbol{x}$ after the deformation $\boldsymbol{u}$.

In our study, we consider a pairwise response function given by:

$$
\boldsymbol{f}\left(\boldsymbol{u}^{\prime}-\boldsymbol{u}, \boldsymbol{x}^{\prime}-\boldsymbol{x}\right)=c \cdot s\left(\boldsymbol{u}^{\prime}-\boldsymbol{u}, \boldsymbol{x}^{\prime}-\boldsymbol{x}\right) \frac{\boldsymbol{y}^{\prime}-\boldsymbol{y}}{\left\|\boldsymbol{y}^{\prime}-\boldsymbol{y}\right\|}
$$

where $c$ is the bond-constant which is defined through a strain energy equivalence with classical theories. Note that the last term in the above equation is the unit vector between $\boldsymbol{x}^{\prime}$ and $\boldsymbol{x}$ after deformation. For a $2 \mathrm{D}$ plane stress system, $c$ is given by [2]:

$$
c=\frac{12 K}{\pi h \delta^{3}}
$$

$K$ is the bulk modulus of the material in question and $h$ is the thickness. Note that bond-based peridynamics restricts Poisson's ratio to $1 / 3$ for a $2 \mathrm{D}$ plane-stress system. As a result, bond-based peridynamics has one unique material constant which [2] chooses to be the bulk modulus. The expression for the pairwise response function satisfies Newton's third law of motion in that $\boldsymbol{f}\left(\boldsymbol{u}^{\prime}-\boldsymbol{u}, \boldsymbol{x}^{\prime}-\boldsymbol{x}\right)=-\boldsymbol{f}\left(\boldsymbol{u}-\boldsymbol{u}^{\prime}, \boldsymbol{x}-\boldsymbol{x}^{\prime}\right)$. This peridynamic formulation also satisfies the balance of linear and angular momentum [2]. One can interpret (3) as the force due to the elongation of a spring.

\subsection{Discretization}

The domain is discretized using a square grid as shown in Figure 1. We assume that the mass of each element is concentrated at the center of each element. As a result, the integral in (1) becomes a sum as given by (5), ignoring body forces.

$$
\rho\left(\boldsymbol{x}_{i}\right) \ddot{\boldsymbol{u}}_{i}\left(\boldsymbol{x}_{i}, t\right)=\sum_{p=1}^{m} \boldsymbol{f}\left(\boldsymbol{u}_{p}-\boldsymbol{u}_{i}, \boldsymbol{x}_{p}-\boldsymbol{x}_{i}\right) V_{p}=\tilde{\boldsymbol{f}}\left(\boldsymbol{x}_{i}\right)
$$

where $m$ is the number of nodes within the horizon of node $i, p$ is the index of a node within the horizon of node $i$, and $V_{p}$ is the volume of node $p$. The horizon is chosen to be three times the mesh spacing as suggested in $[3,4]$.

The integral over $H_{x}$ becomes a sum of forces between $\boldsymbol{x}$ and each node in its family. One can view these forces as resulting from a stretched spring with spring constant $c$. With this interpretation, discretized PD can be seen as a nonlocal spring-lattice model. Additional information on PD can be found in [1,2]. Useful pseudocode for programming PD can be in found in [5].

\subsection{Surface Correction}

The number of bonds between nodes near the edge of a domain is reduced since the distances between these nodes and the boundary of the domain are less than the horizon as shown in Figure 2. The $x$-plane refers to a plane of constant $x$ and the $y$-plane refers to a plane of constant $y$ for point $\boldsymbol{x}$. The volumes 

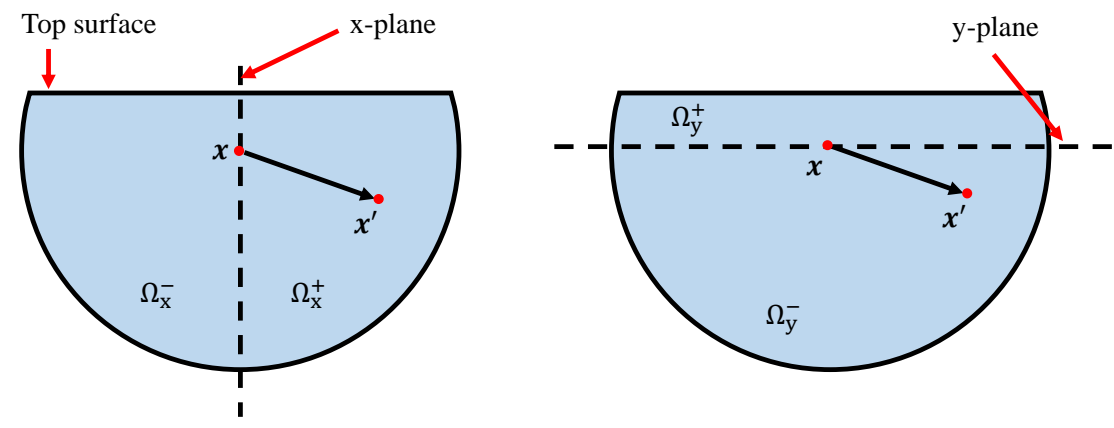

Figure 2: Geometry for Surface Correction

$\Omega_{x}^{+}, \Omega_{x}^{-}$and $\Omega_{y}^{+}, \Omega_{y}^{-}$are defined as the points within the horizon of $\boldsymbol{x}$ that are divided by either the $x$ or $y$-plane. Since the problem below relies on data at a surface, a correction factor must be used $[6,7]$.

(5) now becomes:

$$
\rho\left(\boldsymbol{x}_{i}\right) \ddot{\boldsymbol{u}}_{i}\left(\boldsymbol{x}_{i}, t\right)=\sum_{p=1}^{m} g_{i p c} \boldsymbol{f}\left(\boldsymbol{u}_{p}-\boldsymbol{u}_{i}, \boldsymbol{x}_{p}-\boldsymbol{x}_{i}\right) V_{p}=\tilde{\boldsymbol{f}}\left(\boldsymbol{x}_{i}\right)
$$

where $g_{i p c}$ is the correction factor for the bond between $\boldsymbol{x}_{p}$ and $\boldsymbol{x}_{i}$.

In order to find $g_{i p c}$ we must first find the components of the force density, $g_{x}(\boldsymbol{x})$ and $g_{y}(\boldsymbol{x})$. These scalars are found by evaluating (1) for a given deformation. Note that $g_{x}$ and $g_{y}$ should be calculated at the location of nodes which require the correction. Also, we should recognize that this methodology is mesh and material dependent. Setting

$$
\boldsymbol{u}(\boldsymbol{x})=\theta\left\{\begin{array}{c}
x \\
-\nu y
\end{array}\right\}
$$

where $\theta$ is a constant displacement gradient, whose value is irrelevant other than the fact that it should be much smaller than one, and $\nu$ is Poisson's ratio. For the applied deformation, the following integral is calculated:

$$
g_{x}(\boldsymbol{x})=\left(\int_{\Omega_{x}^{+}} \boldsymbol{f}\left(\boldsymbol{u}^{\prime}, \boldsymbol{u}, \boldsymbol{x}^{\prime}, \boldsymbol{x}\right) \mathrm{d} V^{\prime}-\int_{\Omega_{x}^{-}} \boldsymbol{f}\left(\boldsymbol{u}^{\prime}, \boldsymbol{u}, \boldsymbol{x}^{\prime}, \boldsymbol{x}\right) \mathrm{d} V^{\prime}\right)_{x}
$$

Note that only the $x$-component of the right hand side is required. Similarly for the $y$-direction, we impose a deformation of

$$
\boldsymbol{u}(\boldsymbol{x})=\theta\left\{\begin{array}{c}
-\nu x \\
y
\end{array}\right\}
$$

and then calculate the force density

$$
g_{y}(\boldsymbol{x})=\left(\int_{\Omega_{y}^{+}} \boldsymbol{f}\left(\boldsymbol{u}^{\prime}, \boldsymbol{u}, \boldsymbol{x}^{\prime}, \boldsymbol{x}\right) \mathrm{d} V^{\prime}-\int_{\Omega_{y}^{-}} \boldsymbol{f}\left(\boldsymbol{u}^{\prime}, \boldsymbol{u}, \boldsymbol{x}^{\prime}, \boldsymbol{x}\right) \mathrm{d} V^{\prime}\right)_{y}
$$

Since (8) and (10) required integrations over $\Omega_{x}^{+}, \Omega_{x}^{-}$and $\Omega_{y}^{+}, \Omega_{y}^{-}$, their values are unequal, which would result in an anisotropic response. Therefore, the force densities calculated from (8) and (10) are compared with values as calculated for a point whose horizon lies entirely within the interior of the discretized domain. This value provides a value to normalize against and is denoted by $g_{\infty}$. Using the deformation in (7),

$$
g_{\infty}=2\left(\sum_{p=1}^{m} \boldsymbol{f}\left(\boldsymbol{u}_{p}, \boldsymbol{u}_{i}=\mathbf{0}, \boldsymbol{x}_{p}, \boldsymbol{x}_{i}=\mathbf{0}\right) V_{p}\right)_{x} \forall \boldsymbol{x}_{p} \in \Omega_{x}^{+}
$$

With these values, we calculate: 


$$
\boldsymbol{g}_{i c}=\boldsymbol{g}_{c}\left(\boldsymbol{x}_{i}\right)=\left\{\begin{array}{l}
g_{\infty} / g_{x}\left(\boldsymbol{x}_{i}\right) \\
g_{\infty} / g_{y}\left(\boldsymbol{x}_{i}\right)
\end{array}\right\}
$$

Then, we find

$$
\boldsymbol{g}_{i p c}=\left\{\begin{array}{l}
g_{i p c x} \\
g_{i p c y}
\end{array}\right\}=\frac{\boldsymbol{g}_{i c}+\boldsymbol{g}_{p c}}{2}
$$

Finally, the correction factor is

$$
g_{i p c}=\left(\left[\frac{n_{x}}{g_{i p c x}}\right]^{2}+\left[\frac{n_{y}}{g_{i p c y}}\right]^{2}\right)^{-\frac{1}{2}}
$$

where $n_{x}$ and $n_{y}$ are given by

$$
\left\{\begin{array}{l}
n_{x} \\
n_{y}
\end{array}\right\}=\frac{\boldsymbol{x}_{p}-\boldsymbol{x}_{i}}{\left\|\boldsymbol{x}_{p}-\boldsymbol{x}_{i}\right\|}
$$

\section{Introduction to Cellular Automata}

Cellular Automata (CA) was first introduced by John von Neumann [8] and adapted to elastodynamics by Leamy et al. $[9,10]$. In rectangular form (see [10] for a triangular variant), CA's governing interior equations are mathematically equivalent to subjecting classical elasticity's governing equation to the central difference finite difference method. However, CA avoids derivation of the governing partial differential equations. CA also yields straight-forward treatment of common boundary conditions based on physical considerations.

CA divides the domain into cells and in our case, the shape of the cells are squares. Each cell's state is dependent on the state of cells that share an edge or vertex with the cell in question. The state of a cell is defined as its deformation and velocity in both the $x$ and $y$ directions. The state of each cell is dependent on the state of the cell and its neighbors in the previous timestep.

In the absence of body and external forces, from the balance of momentum, given as an example, the velocity in the $y$ direction for an element in the $(i, j)$ position, reproduced from [9], is given as:

$$
\begin{aligned}
\rho(\Delta x \Delta y \Delta z) \dot{v}_{y}(i, j)= & (\Delta x \Delta y \Delta z)\left[\frac{\lambda+2 \mu}{\Delta y}\left(\frac{u_{y}(i, j+1)-u_{y}(i, j)}{\Delta y}-\frac{u_{y}(i, j)-u_{y}(i, j-1)}{\Delta y}\right)\right. \\
& \left.+\frac{\lambda}{2 \Delta y}\left(\frac{u_{x}(i+1, j+1)-u_{x}(i-1, j)}{2 \Delta x}-\frac{u_{x}(i+1, j-1)-u_{x}(i-1, j-1)}{2 \Delta x}\right)\right] \\
& +(\Delta x \Delta y \Delta z) \mu\left[\frac{1}{\Delta x}\left(\frac{u_{y}(i+1, j)-u_{y}(i, j)}{\Delta x}-\frac{u_{y}(i, j)-u_{y}(i-1, j)}{\Delta x}\right)\right. \\
& \left.+\frac{1}{2 \Delta x}\left(\frac{u_{x}(i+1, j+1)-u_{x}(i+1, j-1)}{2 \Delta y}-\frac{u_{x}(i-1, j+1)-u_{x}(i-1, j-1)}{2 \Delta y}\right)\right]
\end{aligned}
$$

where $\Delta x, \Delta y, \Delta z$ are the grid spacing, $\rho$ is mass density, $\lambda$ and $\mu$ are Lame's constants. The deformation in the $x$ and $y$ direction is $u_{x}$ and $u_{y}$ respectively. The velocity in the $y$ direction is $v_{y}$ and the overdot signifies a derivative with respect to time. Note that $\rho, \lambda$ and $\mu$ can vary in space. (16) is in the form of a finite difference equation which one can derive partial differential equations.

Traction boundary conditions, in CA, require an additional layer of cells for which the defined stress is prescribed. Displacement boundary conditions assign the displacement directly. For more information on this topic one should consult [9]. 


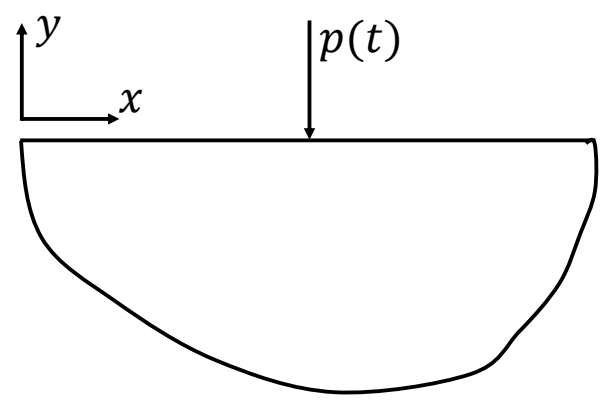

Figure 3: Lamb's Problem

\section{Problem Parameters}

The problem considered here is a half-space subjected to a normal impulse-type line load, classically known as Lamb's Problem, see Figure 3. This problem is independent of the $z$-axis, out of plane axis, and is simplified to a $2 \mathrm{D}$ plane-stress problem.

This is a classical problem that has been thoroughly studied analytically and experimentally $[11,12,13$, 14] and includes many characteristics with which to evaluate CA and PD with. They include the pressure $(\mathrm{P})$, shear $(\mathrm{S})$ and Rayleigh (R) waves.

Dally, et al [11] studied this problem experimentally and results are reproduced below. The material modeled is CR-39 as it is used in [11] for their photoelastic properties. CR-39 has an elastic modulus of 3.85 GPa (559 ksi), Poisson ratio of $1 / 3$ and mass density $1300 \mathrm{~kg} / \mathrm{m}^{3}$. The experimental study used an explosive charge as its input which cannot be exactly reproduced for our numerical study. Therefore, consistent with [11], the approximate loading input for the numerical methods is a triangular pulse as shown in Figure 4.

Dally originally compared his experimental data with predictions from the equations of classical continuum mechanics and elasticity which is referred to as 'theoretical' below. Superposition of results given by $[15,16]$ and assuming $\sigma_{y y}=0$ and $\sigma_{x y}=0$ on the top surface yields the equations given by Dally [11] which are reproduced below.

$$
\begin{gathered}
\left.\sigma_{x x}(x, t)\right|_{y=0}=0 ; \quad c_{1} t=\sqrt{3} c_{2} t \leq x \\
\left.\sigma_{x x}(x, t)\right|_{y=0}=-\frac{2}{3 \pi c_{2}} \int_{\sqrt{3}}^{c_{2} t / x} F(\eta) \dot{p}\left(t-\frac{\eta x}{c_{2}}\right) \mathrm{d} \eta ; \quad c_{2} t \leq x \leq c_{1} t \\
\left.\sigma_{x x}(x, t)\right|_{y=0}=-\frac{2}{3 \pi c_{2}} \int_{\sqrt{3}}^{1} F(\eta) \dot{p}\left(t-\frac{\eta x}{c_{2}}\right) \mathrm{d} \eta ; \quad c_{R} t \leq x \leq c_{2} t \\
\left.\sigma_{x x}(x, t)\right|_{y=0}=-\frac{2}{3 \pi c_{2}} \int_{\sqrt{3}}^{1} F(\eta) \dot{p}\left(t-\frac{\eta x}{c_{2}}\right) \mathrm{d} \eta+\frac{1}{3 c_{R}} \dot{p}\left(t-\frac{x}{c_{R}}\right) ; \quad 0 \leq x \leq c_{R} t
\end{gathered}
$$

Pressure, shear and Rayleigh waves are represented by $c_{1}, c_{2}$ and $c_{R}$ respectively. For CR-39, the wavespeeds are $c_{1}=1826 \mathrm{~m} / \mathrm{sec}, c_{2}=1054 \mathrm{~m} / \mathrm{sec}$ and $c_{R}=969 \mathrm{~m} / \mathrm{sec}$. The loading function is given by $p(t)$ and the overdot signifies the derivative with respect to time. $F(\eta)$ is given below.

$$
F(\eta)=\frac{3 \eta^{2}\left(0.5-\eta^{2}\right) \sqrt{\left(1-\eta^{2}\right)\left(\eta^{2}-\frac{1}{3}\right)}}{2\left(\eta^{2}-0.25\right)\left(\eta^{2}-\frac{3-\sqrt{3}}{4}\right)\left(\frac{3+\sqrt{3}}{4}-\eta^{2}\right)}
$$

In this paper, experimental and theoretical boundary stress results were compared with CA and the boundary displacements were compared between PD and CA. The pressure wave amplitude decay plot is also given. 


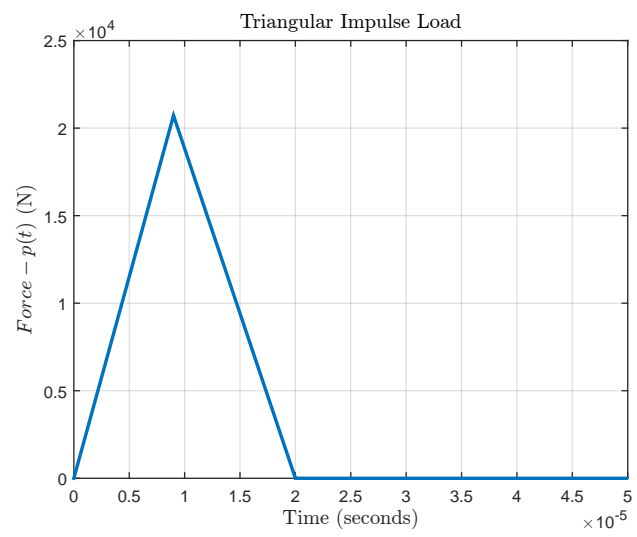

Figure 4: Triangular Impulse Load

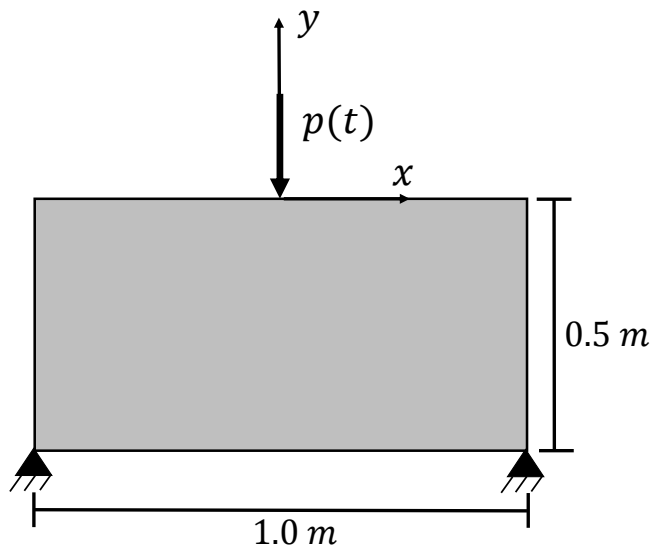

Figure 5: Model Geometry 


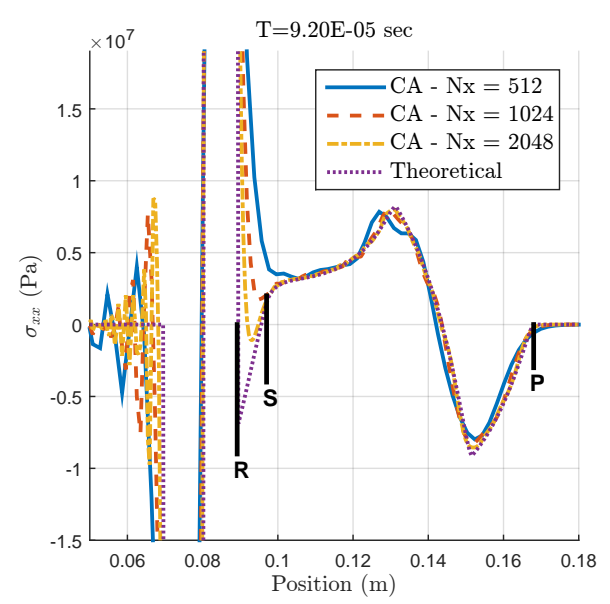

(a)

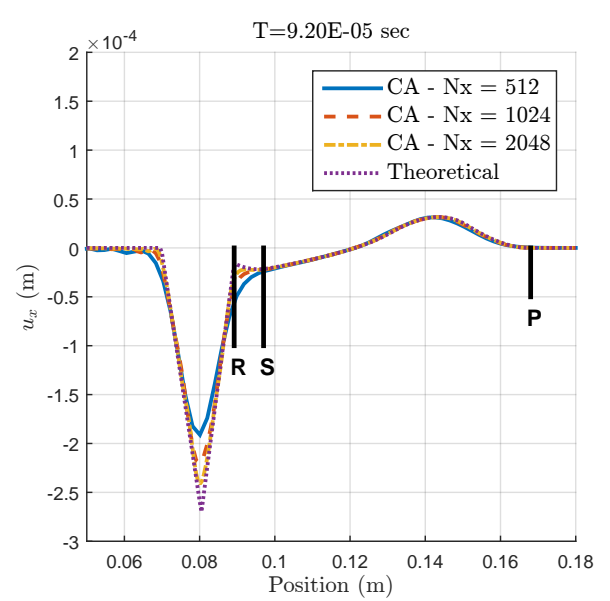

(b)

Figure 6: At 92 $\mu \mathrm{sec}$ : CA Mesh Convergence

\section{Model Geometry}

The domain of the simulation is $1.0 \mathrm{~m}$ wide, $0.5 \mathrm{~m}$ high with a thickness of $6.655 \mathrm{~mm}$ (consistent with [11]). The impulse is applied at the origin of the system, midpoint of the top edge, as shown in Figure 5. Figure 4 shows the applied impulse load with a pulse width of $20 \mu \mathrm{sec}$ and a maximum amplitude of $20.7 \mathrm{E} 3$ $\mathrm{N}$. This amplitude was chosen as it closely reflects the amplitude of the pressure wave at $92 \mu \mathrm{sec}$. The simulation end time is $208 \mu \mathrm{sec}$ and with a pressure wavespeed of $1826 \mathrm{~m} / \mathrm{sec}$, we expect the wave to travel about $0.38 \mathrm{~m}$, well within the size of the domain. As a result, edge effect or reflections are not of concern. The domain was restricted from rigid body motion by fixing the bottom left and bottom right corners. The domain is discretized with 1024 nodes in the $x$-direction and 512 nodes in the $y$-direction, therefore grid spacing is about $0.977 \mathrm{~mm}$. There is one node in the $z$-direction in order to simulate the 2D formulation. With this grid spacing a timestep of $0.125 \mu \mathrm{sec}$ was chosen. Given below is a convergence study. Since the system is symmetric with respect to the loading axis, only the right half of the simulation is presented.

Following with [5], the time-marching algorithm used is the velocity-Verlet method which is reproduced in Table 1. At point $i$, the mass density is represented by $\rho_{i}$. For particle $i$ at timestep $n$, the force density is given by $\tilde{\boldsymbol{f}}_{i}^{n}$, velocity is $\boldsymbol{v}_{i}^{n}$ and deformation is represented by $\boldsymbol{u}_{i}^{n}$.

\begin{tabular}{ll}
\hline Step 1: & $\boldsymbol{v}_{i}^{n+1 / 2}=\boldsymbol{v}_{i}^{n}+\frac{\Delta t}{2 \rho_{i}} \tilde{\boldsymbol{f}}_{i}^{n}$ \\
\hline Step 2: & $\boldsymbol{u}_{i}^{n+1 / 2}=\boldsymbol{u}_{i}^{n}+\Delta t \boldsymbol{v}_{i}^{n+1 / 2}$ \\
\hline Step 3: & Update pairwise response function \\
\hline Step 4: & $\boldsymbol{v}_{i}^{n+1}=\boldsymbol{v}_{i}^{n+1 / 2}+\frac{\Delta t}{2 \rho_{i}} \tilde{\boldsymbol{f}}_{i}^{n+1}$ \\
\hline
\end{tabular}

Table 1: Velocity Verlet Method

\section{Convergence Study}

For the given convergence study, the grid spacing in the $x$ and $y$ directions are equal and the thickness was as given above. The ratio between the grid spacing, $\Delta x$ and the time step, $\Delta t$ was held constant. Three different mesh densities were considered to determine if the system converged for both PD and CA. The domain was held fixed with a $1.0 \mathrm{~m}$ wide and $0.5 \mathrm{~m}$ tall. The number of nodes along the width is denoted by $N_{x}$. The grid spacing and timestep information is given in Table 2. Each model was evaluated at $92 \mu$ sec, the approximate halfway point of the simulation. Theoretical results are also provided for reference. 


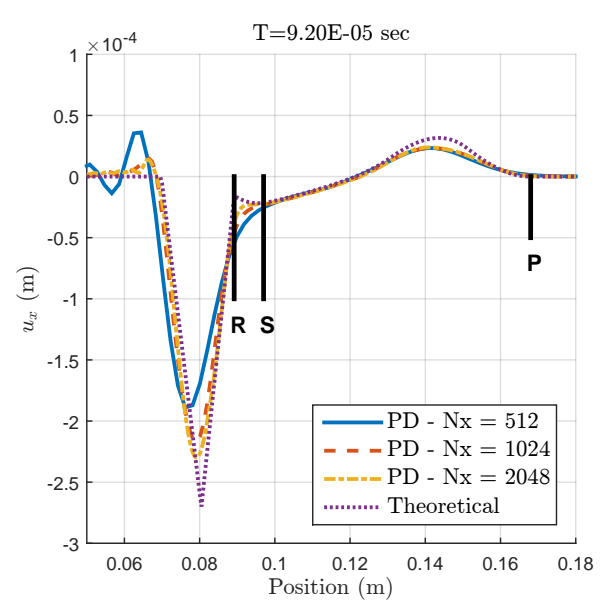

(a)

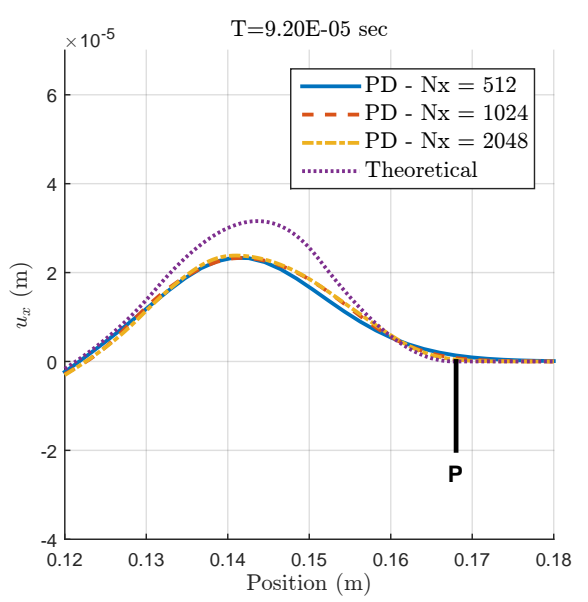

(b)

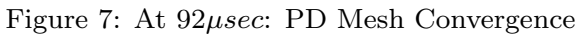

\begin{tabular}{ccc}
$\mathrm{Nx}$ & $\Delta x(\mathrm{~mm})$ & $\Delta t(\mu \mathrm{sec})$ \\
\hline 512 & 1.95 & 0.25 \\
1024 & 0.977 & 0.125 \\
2048 & 0.488 & 0.0625
\end{tabular}

Table 2: Grid Spacing and Timesteps

\subsection{Cellular Automata}

Figure 6 shows the results of the convergence study where Figure 6 a shows the $x x$-component of stress and Figure $6 \mathrm{~b}$ shows the $x$-component of displacement. As seen in both figures, the compressive-loading pressure wave for the given mesh densities overlap and there no meaningful improvement to the solution. However there is some deviation between the stress results in the trailing tensile-unloading pressure wave between $0.1 \mathrm{~m}$ and $0.13 \mathrm{~m}$. The coarser meshes show some high-frequency oscillations and the fine mesh is smoother. However, the displacement results show no such issues, most likely due to the oscillation's small amplitude. Also, the finer mesh is better able to predict the shape of the shear wave compared to the coarser meshes. The coarse meshes seem to blur the interface between the shear and surface waves. The solution predicted by CA seems to approach the theoretical solution as the mesh is refined. Nevertheless, a grid spacing of $\Delta x=0.977 \mathrm{~mm}$ or $N_{x}=1024$ is sufficient.

\subsection{Peridynamics}

Figure 7 shows the results of the convergence study where Figure 7 a shows the $x$-component of displacement at the surface and Figure 7b takes a closer look at the pressure wave. As the mesh density increases, holding $\delta=3 \Delta x$, we can see that there is marginal improvement to the shape of the pressure wave. We also note that the amplitude of the Rayleigh wave increases as the mesh is refined. Looking closely at the figures, we can note that, as the mesh is refined, the speed of the waves seems to change. Figure $7 \mathrm{~b}$ shows that the front edge of the $\mathrm{P}$ wave moves toward the theoretical solution as the mesh is refined. A similar phenomenon is seen in the Rayleigh wave as it quickens as the mesh is refined.

However, it should be noted that the amplitude of the $\mathrm{P}$ wave does not converge to the theoretical value. This may be due to several factors. One of which may be the surface correction methodology used here. There are several proposed methodologies to correct for a free surface in PD and it has yet to be determined which is preferred $[2,6,17]$. Another source of error is the volume correction procedure [2] which does not take into account the entire volume encompassed by the horizon or the error may be due to some unknown factor. 
While increasing $N_{x}$, the number of nodes along the width, from 512 to 1024 shows improvement in the location and magnitude of the Rayleigh wave, further increasing $N_{x}$ to 2048 does not improve the results significantly. As a result, $N_{x}=1024$ is sufficient.

It should be noted that peridynamics is capable of modeling finite deformations as the vector in equation (3) is in the deformed direction. Knowing this and the fact that increasing the mesh density can cause excessive local deformation near a point load, peridynamics could produce a result with considerable nonlinear terms that would not reflect the results of the theoretical, experimental or CA models. As a result, as the mesh was refined, the point load was spread over several nodes to maintain the area subjected to excitation. For $N_{x}=1024$, the load was spread over 4 nodes. To be consistent, the CA model was subject to the same loading conditions. Since we are only looking at the right side of the model, the $x=0$ position is defined as the right most node subjected to loading.

\section{Experimental Results}

It should be noted that the experimental and theoretical results deviate from each other. Dally contributes this discrepancy to several reasons. One reason is that the experiment was performed in a 3D setting and the plane-stress assumption may not capture some effects. Also, the material in question, like all materials, has some internal damping/friction which the theoretical model does not take into account. Furthermore, the existence of wave dispersion may help explain as to why there was excessive wave attenuation in the experiment. Also note that the theoretical applied load is a normal triangular impulse-load, which is only an approximation to the explosive loading that Dally used in his experiments. The explosive load is likely to include a force parallel to the free surface in addition to the normal load.

Dally contributes the significant difference of the $\mathrm{S}$ and $\mathrm{R}$ waves between the theoretical and experimental results due to damage of the CR-39 sample. As we will see below, the P-wave is initially compressive and it's tail is tensile which resulted in some localized fracture. Dally believed that this fracturing caused significant amplitude decrease in the $\mathrm{S}$ and $\mathrm{R}$ waves. For a more detailed discussion, the reader is referred to Dally [11].

\section{Model Results}

\subsection{Cellular Automata Results}

Figures 8a, 9a, 10a, and 11a plot the $x x$-component of stress on the surface for CA. Note that the wavespeed in the CA model is within $2 \%$ of the theoretical value. In each of these figures, vertical lines are placed at the position where the pressure $(\mathrm{P})$, shear $(\mathrm{S})$ and Rayleigh $(\mathrm{R})$ waves are expected to begin. Figure 12a provides the minimum and maximum stress amplitude attenuation due to the pressure wave. Each of these figures include analytical and experimental results [11].

The photoelastic experimental results at $107 \mu \mathrm{sec}$ are given in Figure 13a and a contour plot of stress predicted by CA is given in Figure 13b. The stress optic law for photoelastic materials is given as:

$$
\sigma_{1}-\sigma_{2}=\frac{N f_{\sigma}}{h}
$$

where $\sigma_{1}$ and $\sigma_{2}$ are principle stresses in the $x$ and $y$ directions, respectively, $N$ is the fringe order, $f_{\sigma}$ is the material fringe value, for CR-39 is $19088.8 \mathrm{~Pa}-\mathrm{m}$ (109 psi-in) and $h$ is the model thickness which is 6.655 $\mathrm{mm}$. The isostress lines given in Figure 13b are equivalent to the photoelastic results.

\subsection{Peridynamic Results}

Figures 8b, 9b, 10b, and 11b plot the surface displacements in the $x$-direction for the PD and CA models. Note that the wavespeed in the PD model is within $2 \%$ of the theoretical value. In each of these figures, vertical lines are placed at the position where the pressure $(\mathrm{P})$, shear $(\mathrm{S})$ and Rayleigh $(\mathrm{R})$ waves are expected to begin. Unfortunately, the results given by [11] are in terms of Cauchy Stress. PD, since it is a nonlocal theory, does not have an equivalent measurement. Therefore, experimental results cannot be directly compared with PD. However, since at the surface $\sigma_{y y}=0$ and $\sigma_{x y}=0$, we can use classical continuum mechanics to find a relationship for the displacement. We find that 


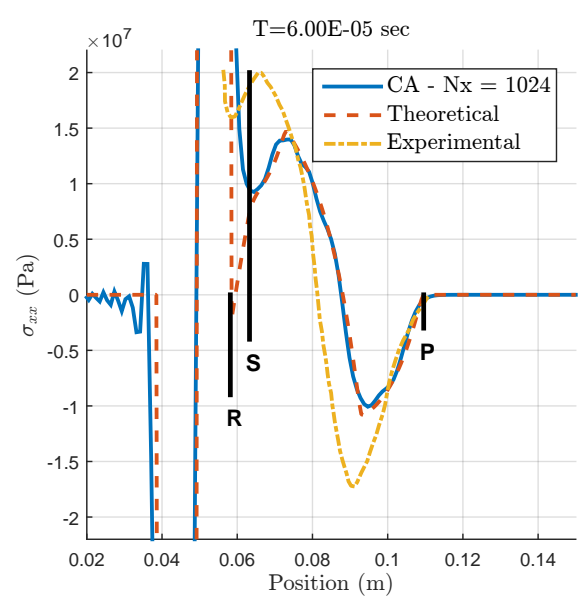

(a)

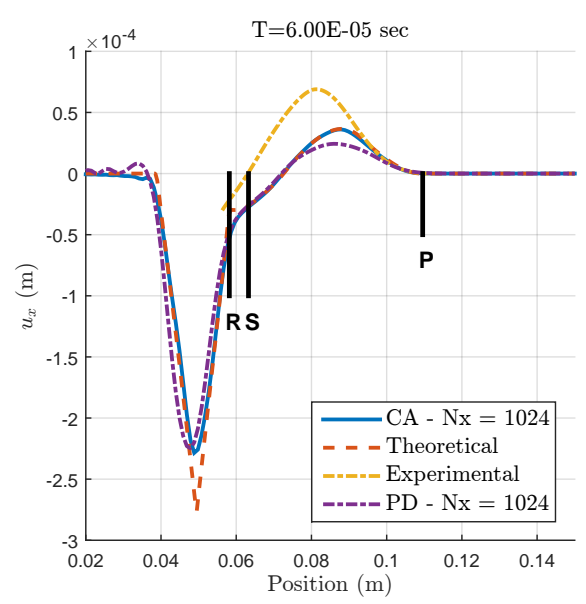

(b)

Figure 8: At 60 $\mu \mathrm{sec}$ : Comparing Theoretical and Experimental with (a) CA Stress, (b) PD and CA Displacements

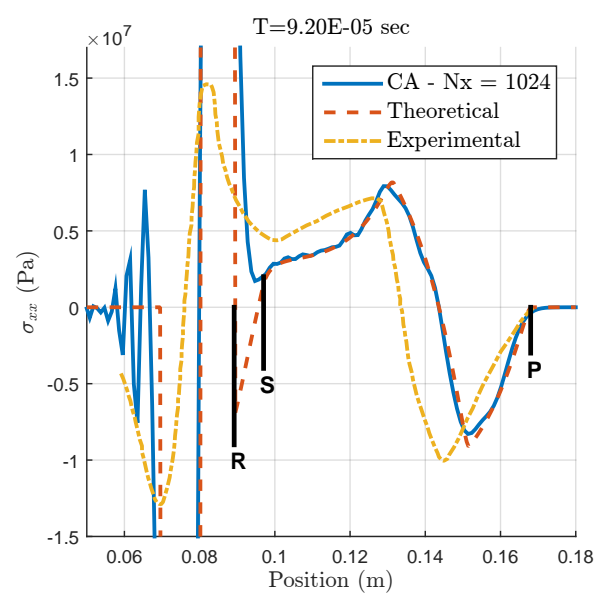

(a)

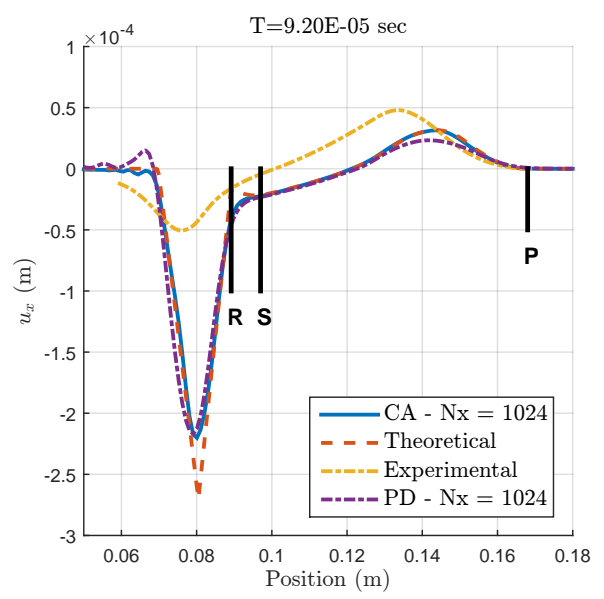

(b)

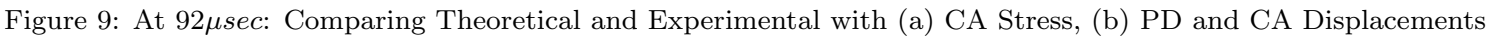

$$
\frac{\partial u_{x}}{\partial x}=\frac{\sigma_{x x}}{2 \mu(1+\nu)}
$$

With the relationship above, we can numerically integrate the experimental results to recover the displacement. Figure $12 \mathrm{~b}$ provides the maximum and minimum displacement amplitude attenuation due to the pressure wave and Rayleigh wave.

Figure 14b is a contour plot of the magnitude of displacements for PD at $107 \mu s e c$ and Figure 14a provides the equivalent plot for CA.

\section{Discussion and Conclusion}

Figures $8,9,10$, and 11, show that the CA-predicted and theoretical results match very well for the initial pressure wave in both shape and location. The amplitude decay of the pressure wave coincides with the theoretical result as seen in Figure 12a. This result is expected since both the theoretical and CA-predicted results are essentially derived from the same governing equations. As shown above, as the mesh is further refined, $\mathrm{CA}$ is better able to predict the location and magnitude of the Rayleigh wave. 


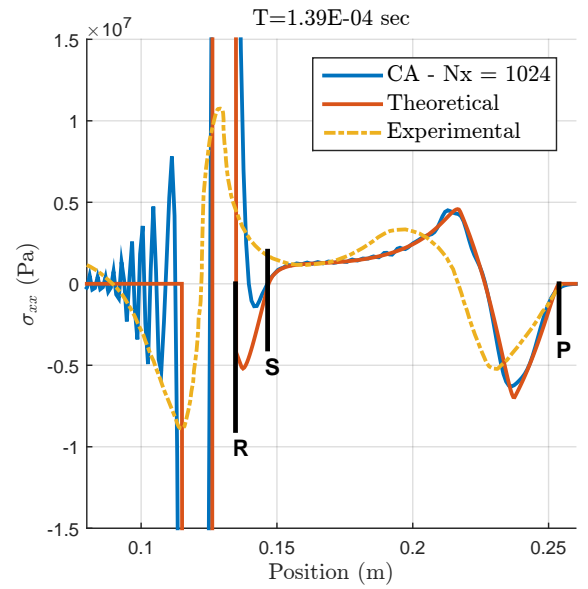

(a)

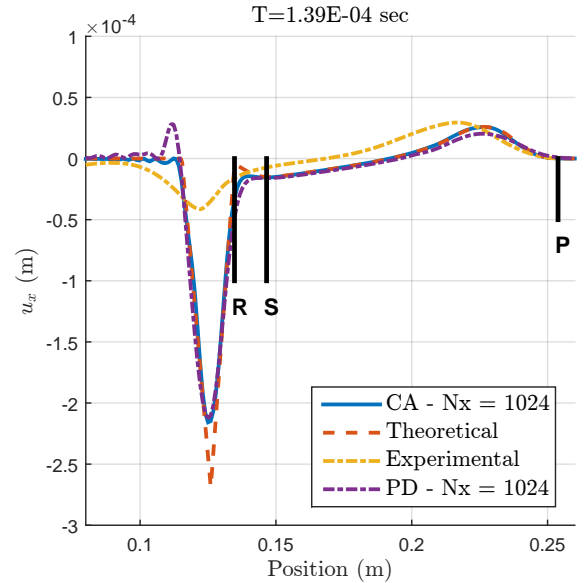

(b)

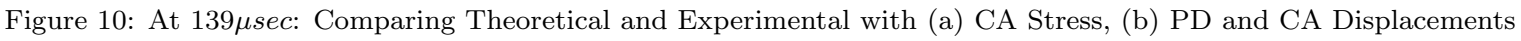

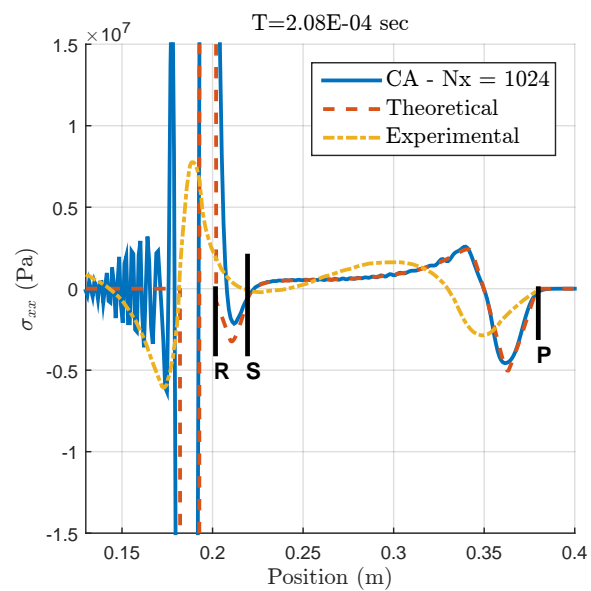

(a)

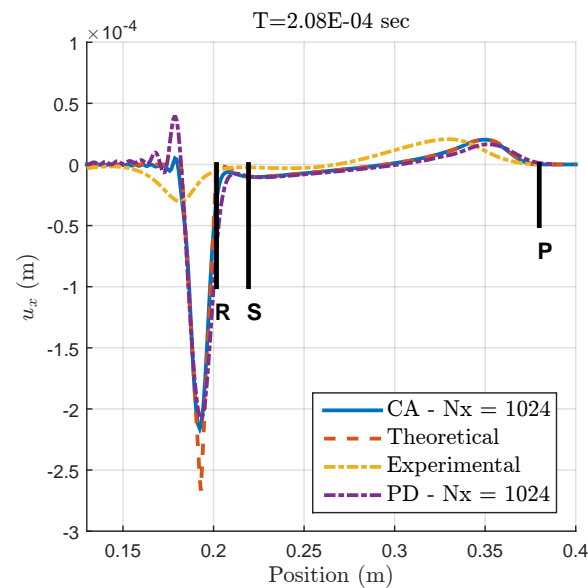

(b)

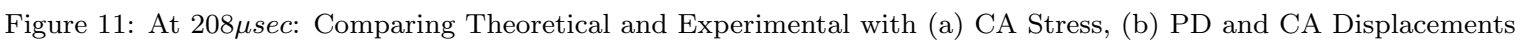
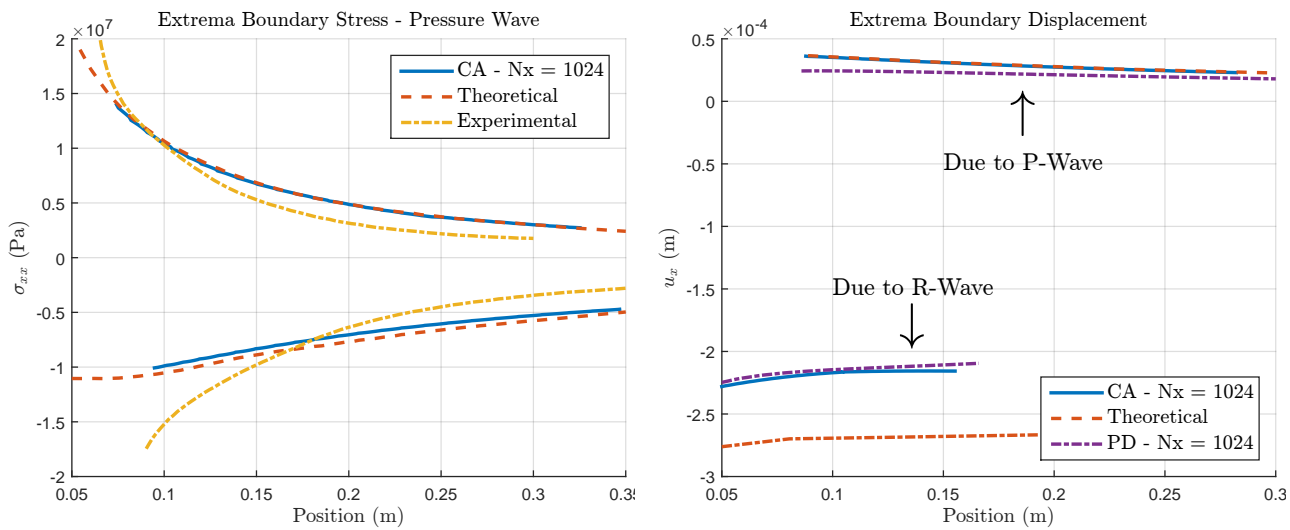

(a) Comparing CA with Experimental and An-(b) Comparing PD and CA Displacement Realytical Results sults

Figure 12: Amplitude Decay of Pressure Wave 


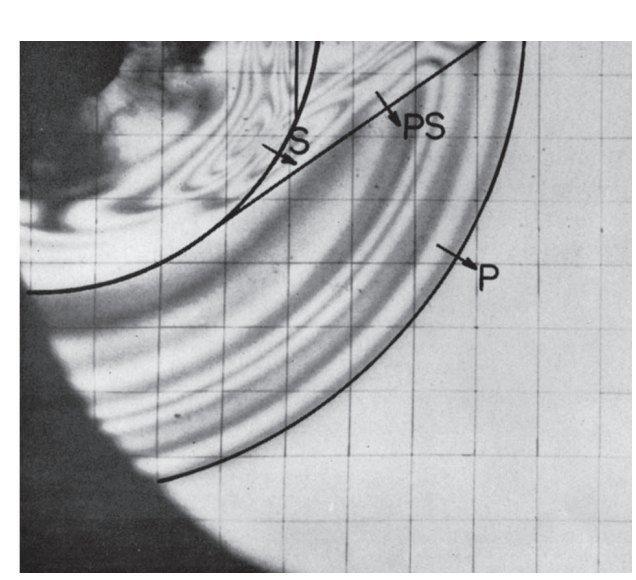

(a) Photoelastic Results from [11]

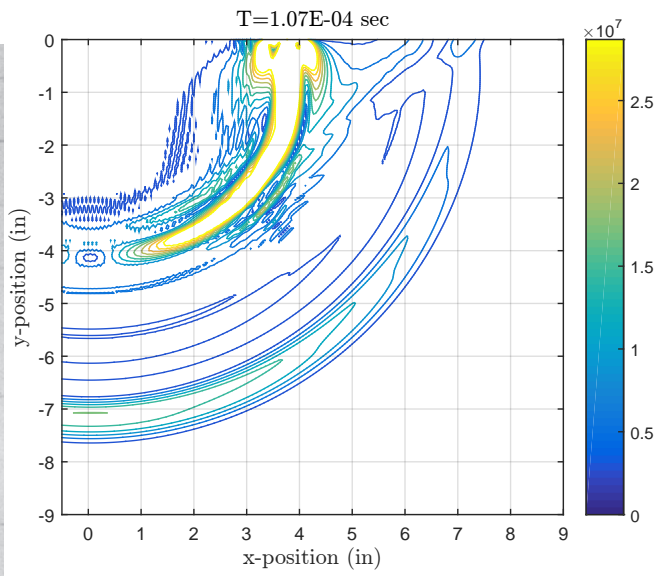

(b) CA Stress Plot

Figure 13: Comparing Photoelastic and CA Stress at $107 \mu \mathrm{sec}$

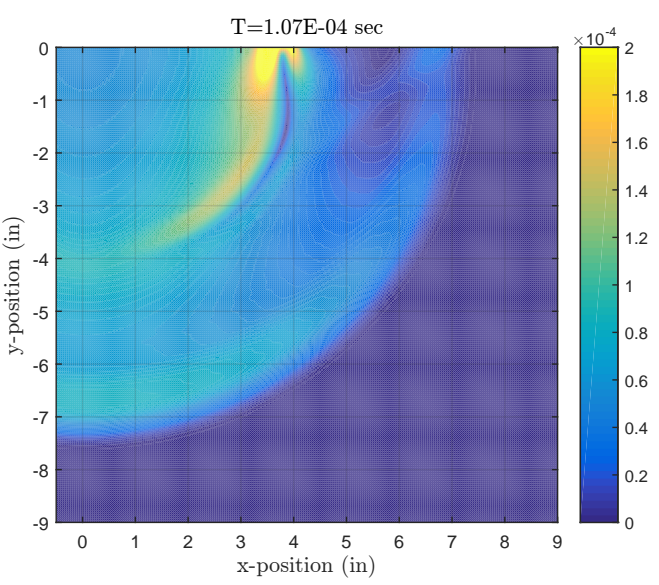

(a) CA Displacement Plot

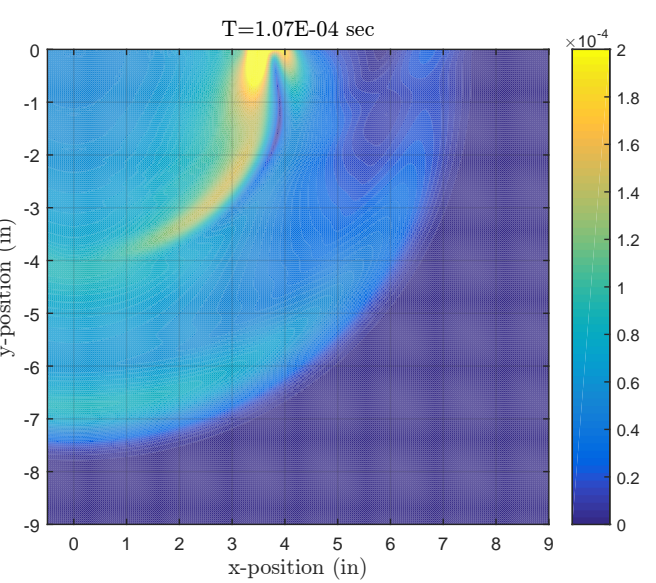

(b) PD Displacement Plot

Figure 14: Comparing CA and PD displacement magnitudes at $107 \mu$ sec 
PD predicted the location and shape of the pressure wave well. However, at earlier times, PD underpredicts the magnitude of the pressure wave. Figure $12 \mathrm{~b}$ provides comparisons between $\mathrm{PD}, \mathrm{CA}$ and theoretical displacement amplitudes due to the $\mathrm{R}$ and $\mathrm{P}$ waves. The differences in $\mathrm{P}$ wave amplitude predicted by $\mathrm{PD}$ and the other models are significant at earlier times. However, the $\mathrm{P}$ wave predicted using PD does not decay as quickly as predicted using the other models. This inconsistency may be due to the surface correction methodology or the volume correction used here. One phenomenon that is seen in PD, and not in $\mathrm{CA}$, is that as the mesh of $\mathrm{PD}$ is refined, wavespeeds change. This phenomenon can be easily seen in the $\mathrm{R}$ wave. As the mesh in refined in $\mathrm{CA}$, the $\mathrm{R}$ wave in Figure $6 \mathrm{~b}$ does not change position. In $\mathrm{PD}$, Figure $7 \mathrm{a}$ shows that the location of the $\mathrm{R}$ wave moves toward the theoretical result as the mesh is refined. Further mesh refinement may show that the PD-predicted results continue converging to the theoretical results. It has been shown that as the horizon decreases, PD converges to classical elasticity [18]. The results shown in Figure 7a support this convergence. However, we see the experimental pressure wave does not follow CA or PD predictions very well. More study is necessary on the relationship of the horizon and wavespeed.

Figure 13 shows that the isostress lines between CA-predicted and experimental values are similar in shape for the initial pressure wave and and are directed towards the surface. However, there is a significant difference in the lines of stress for the shear wave. Additionally, Figure 14 shows that there is excellent agreement between CA and PD throughout the body. Looking carefully at Figures 13 and 14, we can also see the existence of a head wave which Dally cites as 'PS'.

We conclude that both the CA and PD predictions agree well with theoretical results. However, neither method's predictions agreed well with experimental results. In particular, the experimental $\mathrm{P}$ wave amplitude began with a larger amplitude but decayed quicker. This is expected as CA, PD and theoretical solutions do not include any friction or dissipative effects. The discrepancy in the $\mathrm{P}$ wave shape and the small magnitude of the Rayleigh Wave, compared with CA, PD and theoretical results, can be attributed due to the triangular impulse load that was used to approximate an explosive load or due to the two-dimensional approximation of the problem. Dally cites the presence of cracking in the sample as a probably source of error. For CA, agreement with theoretical results is expected since the formulation of CA is mathematically equivalent to the central difference finite difference method. We expected that PD predictions would agree better with experimental results due to its ability to handle finite deformations. Further investigation is needed.

\section{Acknowledgments}

This work was partially supported by the NSF under grants CMMI-1030940 and IIP-1362146 (I/UCRC on Novel High Voltage/Temperature Materials and Structures).

\section{References}

[1] S.A. Silling, Reformulation of Elasticity Theory for Discontinuities and Long-Range Forces, J. Mech. Phys. Solids, 48, (2000), 175-209.

[2] E. Madenci, E. Oterkus, Peridynamic Theory and its Applications, Springer, New York-U.S.A., 2014.

[3] P.N. Demmie, S.A. Silling, An Approach to Modeling Extreme Loading of Structures using Peridynamics, J. of Mech. of Mater. and Structures, 2, (2007) 1921-1945.

[4] W. Liu, J.W. Hong, Discretized Peridynamics for Linear Elastic Solids, Comput. Mech., 50, (2012), 579-590.

[5] M.L. Parks, P. Seleson, S.J. Plimpton, R.B. Lehoucq, S.A. Silling,Peridynamics with LAMMPS: A User Guide v0.2 Beta, Sandia National Laboratories, 2010-5549, (2010).

[6] B. Kilic, E. Madenci, Coupling of Peridynamic Theory and the Finite Element Method, J. Mech. Mater. Structures, 5, (2010).

[7] S.A. Silling, E. Askari, A Meshfree Method Based on the Peridynamic Model of Solid Mechanics, Comput. Struct., 83, (2005), 1526-1535. 
[8] J. von Neumann, The Theory of Self-reproducing Automata, University of Illinois Press, 1966.

[9] M.J. Leamy, Application of Cellular Automata Modeling to Seismic Elastodynamics, Int. J. Solids Struct., 45, (2008), 4835-4849.

[10] R.K. Hopman, M.J. Leamy, Arbitrary Geometry Cellular Automata for Elastodynamics, J. Appl. Mech., 78, (2011).

[11] J.W. Dally, S.A. Thau, Observations of Stress Wave Propagation in a Half-plane with Boundary Loading, Int. J. Solids Structures, 3, (1967) 293-308.

[12] K.F. Graff, Wave Motion in Elastic Solids, Dover Publications, INC., New York-U.S.A., 1991.

[13] E. Kausel, Lamb's Problem at its Simplest, Proc. R. Soc. A, 469, (2013).

[14] H. Lamb, On the Propagation of Tremors over the Surface of an Elastic Solid, Phil. Trans. R. Soc. Lond. A, 203, (1904).

[15] Y.C. Fung, Foundations of Solid Mechanics, Prentice-Hall, 1965.

[16] J.W. Sherwood, Elastic Wave Propagation in a Semi-Infinite Solid Medium, Proc. Phys. Soc., Lond., 71, (1958).

[17] W. Gerstle, N. Sau, S. Silling, Peridynamic Modeling of Plain and Reinforces Concrete Structures, 18th Int. Conf. on Struc. Mech. in Reactor Tech., (2005).

[18] S.A. Silling, R.B. Lehoucq, Convergence of Peridynamics to Classical Elasticity, J. Elast., 93, (2008), $13-37$. 